C2008 IEEE. Personal use of this material is permitted. However, permission to reprint/republish this material for advertising or promotional purposes or for creating new collective works for resale or redistribution to servers or lists, or to reuse any copyrighted component of this work in other works must be obtained from the IEEE. 


\title{
Quantifying the Numeric and Linguistic Magnitude of Perceived Risk in E-Commerce Interactions for RDSS
}

\author{
Omar K. Hussain, Elizabeth Chang, Farookh K. Hussain and Tharam S. Dillon \\ Digital Ecosystems and Business Intelligence Institute \\ Curtin University of Technology, Perth, Australia \\ e-mail: Omar.Hussain@ postgrad.curtin.edu.au
}

\begin{abstract}
In an e-commerce business interaction, the risk assessing agent by analyzing the possible level of perceived risk beforehand can make an informed decision of its future course of interaction with an agent. The perceived risk in the context of an e-commerce business interaction is a multidimensional construct which is the combination of its subcategories. In this paper we propose a methodology by which the risk assessing agent can ascertain the numeric and linguistic level of perceived risk in an interaction by combining its different subcategories.
\end{abstract}

Index Terms-Perceived risk, Performance risk, Financial risk, Possibility theory and Fuzzy sets.

\section{INTRODUCTION}

The rapid pace of development of the internet for facilitating e-commerce interactions has provided users with sophisticated technologies which eases the process of carrying out their activities and the ability to complete their tasks in less time, thus reducing the delays associated with the conventional method of interactions and at the same time boosting their efficiency as well as helping the businesses to improve their sales, productivity and economy. The architectures present for the users to carry out their activities are namely the client-server, peer-to-peer, grid and web-service architectures. But as mentioned in Chang et al. [1] 'The dynamic, open and convenient web environment not only boosts business potential and the economy but also creates concerns of security, trust, privacy and risks'. So the users before utilizing the provided facilities to their advantage, should consider and analyse beforehand these aspects in order to make sure that they achieve what they desire, or get the maximum output in their interactions. Business-toConsumer (B2C) and Consumer-to-Consumer (C2C) are the two interaction facilitating architectures widely utilized to facilitate e-commerce interactions. These architectures are quite different from the centralized ones, as in such interactions the control between the agents or between the interacting partners is within themselves as opposed to between a server and the consumers in a centralized interaction. The demand of $\mathrm{B} 2 \mathrm{C}$ or $\mathrm{C} 2 \mathrm{C}$ type interactions is expected to grow because of their robust nature [2-6]. But apart from all the advantages provided which is resulting in its fast adoption among the users, some important perceptions to consider for the user in such interactions is the notion of trust and risk, i.e. taking into consideration the factors which helps to make the interactions over this paradigm safe secure and an informed one, as an agent in such environments has to make its own decisions and has to be responsive and proactive in that. Trust and Risk are the two concepts associated with an interaction, which are important to consider and which complement each other for the initiating agent to make an informed decision of its future course of action with an agent. Due to the importance of these concepts, there have been various conceptualizations of trust and risk according to the discipline in which they are being discussed in [1, 7-13]. As their conceptualization, the way trust and risk in an interaction are quantified and expressed also varies. Trust in an interaction can be expressed in terms of belief or in terms of probability in an interaction, whereas risk in an interaction is best understood when expressed in terms of probabilities under conditions of uncertainty, which tends towards expressing the possible loss in an interaction. As both these concepts are expressed in terms of probability, it may be possible that they both express the probability related of a certain event, but the type of outcome that each concept tries to express are in contrast to each other. In this sense both trust and risk are opposite concepts to each other. By expressing trust and risk as probabilities in an interaction, the magnitude of effect that they have on the expressed outcomes can also be determined according to their probability of occurrence. In other terms, trust and risk are two concepts whose evaluation would give two contrast perspectives of occurrence of an event, and their way of analysis and outputs achieved from evaluating them are not just converse of each other, but would provide various insight and would give solutions to the various doubts which rise in the initiating agent's mind before the interaction.

Various approaches have been proposed in the literature by which the level of trust in an e-commerce business interaction is ascertained based on which the initiating agent decides on its future course in the interaction. But none of those approaches consider the level and degree of perceived risk along with the level of trust while decision making. By analysing the perceived risk, the risk assessing agent can determine beforehand whether or not it will achieve its desired outcomes and the associated consequences to it in achieving those, in interacting with a risk assessed agent. Based on this analysis the risk assessing agent can determine beforehand the possible direction in which its interaction might head, if it interacts with that risk assessed agent. Risk analysis is important in influencing the behaviour of the risk assessing agent in the interaction, because there is a whole body of literature based in rational economics that argues that the decision to buy is based on the risk-adjusted 
cost-benefit analysis [14]. Thus it commands a central role in any discussion related to a transaction. In order for the initiating agent to consider all the possible scenarios which making an interacting decision with another agent, in this paper we propose a methodology by which it can determine the level and magnitude of perceived risk that could be present in its interaction with the other agent. This paper is organized into 8 sections. In section 2 we define perceived risk according to its interpretation in e-commerce business interactions. In section 3 - 4 we propose a methodology for the risk assessing agent to ascertain the numeric level of perceived risk in the interaction. In section $4-7$ we propose a methodology for the risk assessing agent to ascertain the linguistic level of perceived risk in the interaction. Finally in section 8 we conclude the paper.

\section{Ascertaining Perceived Risk In E-Commerce BUSINESS INTERACTION}

We define perceived risk in e-commerce business domain interactions as the likelihood that the risk assessing agent might not achieve its desired outcomes of the interaction due to the risk assessed agent not acting as expected according to its expectations, in the given context and time once the interaction begins resulting in the loss of investment and resources involved in the interaction. Based on the definition we classify the perceived risk in an e-commerce business interaction into two subcategories, namely:

- Performance risk; and

- Financial risk.

Performance risk represents the likelihood and threat of the risk assessed agent in not providing the risk assessing agent with what it desires in the time period of its interaction, resulting in the negative outcome or failure in the interaction. Financial risk represents the degree and magnitude of loss to the risk assessing agent in the interaction due to the performance risk of the risk assessed agent. The risk assessing agent in order to ascertain the perceived risk in interacting with a risk assessed agent has to determine the above mentioned subcategories according to the context and criteria of its interaction. We have defined a methodology in $[15,16]$ by which the risk assessing agent can ascertain the magnitude and levels of performance risk and financial risk in interacting with a risk assessed agent according to the characteristics of its future interaction with it. Due to space limitation we will not be discussing it in this paper.

The risk assessing agent after determining the performance risk and financial risk in interacting with a risk assessed agent should combine them in order to ascertain the level and magnitude of perceived risk in the interaction. The approach that we utilize to determine the magnitude and level of perceived risk in the interaction is two fold. The first part of the approach deals in ascertaining the magnitude of perceived risk in the interaction numerically by using possibility theory. The second part of the approach ascertains the level of perceived risk in the interaction linguistically by using a fuzzy system. The motive for representing the perceived risk in the interaction numerically and linguistically is due to the fact that the risk assessing agent ascertains the perceived risk in interacting with a risk assessed agent, for better decision making, before initiating an interaction with it. Hence it is logical that there is uncertainty in the risk assessing agent's mind while determining the level of perceived risk; as it is being determined in an interaction which is going to be held in a future period of time, in which nothing is certain. In other terms the perceived risk is being determined in an interaction which is yet to be carried out, and that interaction will have a specific outcome/s but there is uncertainty as to exactly which outcome/s will occur from the likely ones. This uncertainty which is present in the risk assessing agent's mind can be classified into two types, as 'ambiguity' and 'vagueness'. We define ambiguity as that uncertainty in the risk assessing agent's mind which represents its inability of identifying the concrete level/s or magnitudes of perceived risk present in interacting with the other agent, whereas vagueness is defined as that type of uncertainty which represents its inability of identifying the degree or likelihood to which those levels will occur in the interaction. In order to alleviate these types of uncertainties from the risk assessing agent's mind, we propose that it calculates the level and magnitude of perceived risk in interacting with a risk assessed agent by using two methods:

1. Numerical Approach (by utilizing Possibility Theory)

2. Linguistic Approach (by utilizing Fuzzy System).

The numerical approach by using possibility theory determines in numeric terms the magnitude or levels of perceived risk in the interaction, along with the possibility of occurrence of those levels. The linguistic approach deals with fuzzy sets and it determines linguistically and semantically the levels of perceived risk along with their likelihood of occurrence in the interaction. By utilizing these two types of approaches to determine the level of perceived risk, the risk assessing agent can alleviate the ambiguity and vagueness in its mind and in turn can make an informed interacting based decision with the risk assessed agent.

\section{Ascertaining The NuMerical LeVel of PERCEIVED RISK IN AN INTERACTION}

The subcategories which the risk assessing agent determines to ascertain the perceived risk in interacting with a risk assessed agent are mentioned earlier. But while ascertaining the perceived level of risk in an interaction, we propose that the risk assessing agent should take into consideration only two if its constituents from the determined subcategories, namely the possible consequences of failure and the loss of investment probability in the interaction. This is because the risk assessing agent considers the effect of the performance risk of the risk assessed agent while determining the financial risk in interacting with it. To avoid considering its effect again and to omit its duplicated impact we propose that the risk assessing agent while determining the perceived risk in interacting with the risk assessing agent should only consider the possible consequences of failure and the loss of investment probability to its resources in interacting with that agent. So the inputs and output while determining the numerical and linguistic level of perceived risk in the interaction can be classified as:

Inputs:

- Possible Consequences of failure to the risk assessing agent in interacting with the risk assessed agent (PCF); 
- Loss in Investment probability to the risk assessing agent in interacting with the risk assessed agent (LOIP).

Output:

- Level of Perceived Risk in the interaction (PR).

While ascertaining the numerical level of perceived risk in the interaction, the possibility distribution of each of the above mentioned constituents is utilized and not their probability distribution. This is to avoid the disadvantage of the probability distribution where the non-zero value must be assigned to an element from its given set or UoD, whose likelihood of occurrence is very high. Further what ever value is assigned to that element effects the probability value to be assigned to the other elements in the $\mathrm{UoD}$, as the sum of all the probability distribution of the elements is constrained to a sum of 1 . In order to alleviate this disadvantage, we use possibility distribution to represent each constituent of risk in an interaction, as in this distribution assigning a likelihood of 1 to an element does not constraint from assigning that likelihood to any other element from its UoD. Further the output, i.e. the magnitude of perceived risk in the interaction too is represented by possibility distribution which does not contain the disadvantages mentioned earlier of the probability distributions. The numerical magnitude of perceived risk in the interaction by using possibility theory is determined mathematically by a relation between:

Perceived Risk $=$ Possible Consequences of Failure $\mathrm{x}$ Loss of Investment Probability

The operator ' $\mathrm{x}$ ' between the two inputs represents convolution. To determine the distribution of each input constituents (PCF and LOIP) and the output function (PR) we need to define the scope within which each of the particular variable exists. The scope or the universe of discourse (UoD) of the input variables and the output variable is defined by the following sets:

Possible Consequences of Failure $(P C F)=\{0,1,2$, $3 \ldots \ldots \ldots 100\}$ where each element has a unit of $\%$.

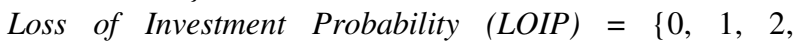
3 ............100\} where each element has a unit of $\%$.

Perceived Risk $(P R)=\{0,1,2,3 \ldots \ldots \ldots \ldots 100\}$ where each element has a unit of $\%$.

To obtain the possibility distribution of an input variable, the likelihood of occurrence of each element from its universe of discourse should be determined. This likelihood of occurrence of an element is termed as the 'degree of evidence' of its outcome represented by ' $m(A)$ ' where ' $A$ ' is an event or an element from the universe of discourse of the input variable ' $\mathrm{X}$ '. From the universe of discourse, those elements with degree of evidence greater than zero are called as the 'focal elements' for the particular input variable. These elements represent the sets from the UoD for that variable upon which the evidence of occurrence focuses and further which will be utilized from that input variable to determine the magnitude of perceived risk in the interaction. The degree of evidence of an element from the UoD should be in the interval between $[0,1]$ and the cumulative sum of the degree of evidence of all the focal elements from the UoD should satisfy the condition [17]:

$$
\sum_{A \in X} m(A)=1
$$

where ' $A$ ' represents the focal elements belonging to the input variable ' $\mathrm{X}$ ',

$m$ (A) represents the degree of evidence of the focal element.

Possibility measures can be represented by a possibility distribution function:

$$
\Pi(X)=\max \{\pi(a) \mid a \in X\}
$$

where $\pi(a)$ is the possibility of the element ' $a$ '.

Equation 2 is repeated for each focal element of ' $\mathrm{X}$ ' to determine its possibility of occurrence. Once the focal elements of the input variables (PCF and LOIP) along with their degree of evidence from their UoD have been determined, they must then be convoluted to determine the perceived risk in the interaction. The focal elements of the output variable function from its defined universe of discourse are determined in the convolution process. The convolution of the possibility distributions is the artesian product of the input variables [17] and is represented by:

$$
X \times Y=\{<x, y>\text { : where } x \in X \text { and } y \in Y\}
$$

where $\langle\mathrm{x}, \mathrm{y}\rangle$ denotes the tuple which represents the artesian product of the input focal elements from their UoD.

The possibility distribution of the focal elements of the resultant output variable as the result of the convolution of the inputs variables is represented by:

$$
\pi(\mathrm{u})=\max \left\{\min \left[\pi_{\mathrm{X}}(\mathrm{x}), \pi_{\mathrm{Y}}(\mathrm{y})\right]\right\}
$$

where $\mathrm{u}$ is the focal element of the output function determined as the artesian product of the inputs $\mathrm{f}(\mathrm{x}, \mathrm{y})$,

$\pi(u)$ is the possibility of focal element ' $u$ ' from the output universe of discourse.

\section{Determining The PossibILITy Distribution OF THE VARIABLES}

In order to ascertain the numerical level of perceived risk in the interaction by using possibility distribution, the risk assessing should first identify the focal elements along with their degree of evidence of the inputs from their universe of discourse which ranges from $\{0 \ldots 100\}$. As discussed in Hussain et al. [16], the risk assessing agent can determine both the constituents namely the loss of investment probability (LOIP) and the possible consequences of failure $(\mathrm{PCF})$ in interacting with the risk assessed agent from the Factual Amount Invested Curve (FAIC) in interacting with it. To determine the focal elements and the possibility distribution of the PCF in the interaction, the risk 
assessing agent should determine the level of extra financial resources from its maximum investment capacity that it has to keep at stake or the level of un-served investment in the interaction while interacting with the risk assessed agent. To achieve that:

- The risk assessing agent should determine the probability mass function (PMF) of the FAIC in interacting with the risk assessed agent. The PMF of the FAIC shows the probability of the net worth of resources that the risk assessing agent has to keep at stake throughout the duration of interacting with the risk assessed agent.

- It should then determine the point on the PMF of the FAIC which represents its maximum investment capacity in the interaction, which is termed as ' $\mathrm{x}$ '.

- From point ' $x$ ' the risk assessing agent should determine the level of extra financial resources that it has to keep at stake or the level of its un-served investment in the interaction.

- From the UoD for the PCF, the focal elements should be chosen according to the measure of step size of the un-served investment in the interaction.

- The risk assessing agent should then determine the degree of evidence (m (A)) of each focal element which represents the level of un-served investment in the interaction. This is determined by taking into consideration the PMF of the particular financial amount from the FAIC and then converting it to possibility distribution, satisfying equation 1 .

- Based on the degree of evidence calculated for each focal element from the UoD, the possibility distribution of the PCF can be determined by using equation 2.

- The LOIP in the interaction, in contrast to the $\mathrm{PCF}$ is a single crisp value in the range of [0-1] which shows the ordinate on the FAIC at the end of its maximum investment capacity in the interaction. But in order to utilize a unified and comparable numerical scale to the two inputs, the range of the LOIP is normalized in the range between 0-100.

- Hence the ordinate of the FAIC after point ' $\mathrm{x}$ ' is taken as focal element for that input from its $\mathrm{UoD}$, to represent the LOIP in the interaction. The degree of evidence of the focal element is taken as 1 .

Once the focal elements and their degree of evidence for each input variable have been determined, the risk assessing agent can then convolute them to determine the magnitude or the focal elements of perceived risk in the interaction from its UoD by using equation 3 . The possibility of occurrence of the focal elements of perceived risk in the interaction can then be determined by using equation 4 .

\section{ASCERTAINING THE LINGUISTICAL LEVEL OF PERCEIVED RISK IN AN INTERACTION}

As discussed before our motive to incorporate the fuzzy system is for the risk assessing agent to ascertain semantically the level of perceived risk in interacting with a risk assessed agent, by combining the different determined constituents of risk present in interacting with it. So an over- view of our proposed fuzzy model with the variables which it takes as inputs and based on that computes an output level of perceived risk is given in figure 1 .

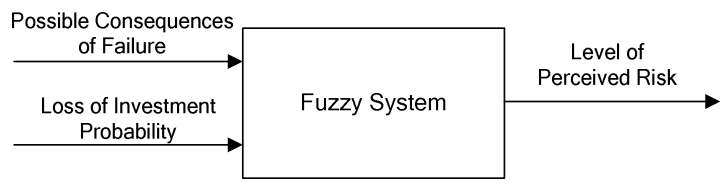

Figure 1: Overview of the fuzzy system to ascertain the level of risk linguistically

Fuzzy systems are mathematical objects modelling the vagueness present in the natural language when the described phenomena do not have sharply defined boundaries. As mentioned in the literature, fuzzy systems were developed to incorporate the concept of partial truth characterized by the fuzziness of the data which yields a more accurate mathematical representation of the perception of truth than that of crisp sets [18]. A fuzzy system models the vague inputs in terms of semantics and transforms them into a mathematical representation of the data to map its output semantics. To achieve that, fuzzy system transforms each input from crisp data to fuzzy sets. So to represent each linguistic variable from crisp data to fuzzy variables, we need to first define the fuzzy sets or the predicates for it. A fuzzy set or predicate is a linguistic phrase that is used as semantic label for representing a part of the variable, which best matches its description. The predicates for a variable should be defined such that it covers the whole numerical range, or the scope in which the variable extends. As discussed earlier, the scope or the numerical range in which the variable extends is called as its universe of discourse (UoD). Each linguistic variable (input and output) has its own universe of discourse depending on the range which it uses to express its function. Once the predicates have been defined for a variable, then the membership function for each of the predicate in that variable should be defined so that they cover its scope or its range of universe of discourse. The membership function is used to transform each crisp input into fuzzy variable, by utilizing the predicates and then determining the degree of qualification or the membership value of the crisp input to those predicates. In the next section we will define the universe of discourse, the predicates and the membership function of each input variable.

\section{DEFINING THE MEMBERSHIP FUNCTIONS FOR THE VARIABLES}

The universe of discourse (UoD) of the input linguistic variable 'Possible Consequences of Failure' ranges from $\{0$, $1,2,3,4,5 \ldots \ldots .100\}$ where each element has unit of $\%$. To classify different fuzzy sets for the input variable 'Consequences of failure' we divide the universe of discourse such that there are 6 predicates in it. The predicates defined for the input variable are: 'Extremely Low', 'Low' 'Low Medium', 'Medium High', 'High' and 'Extremely High'. The membership function of the linguistic variable 'Possible 
Consequences of Failure' in the trapezoidal curve such that it is of the form as shown in figure 2.

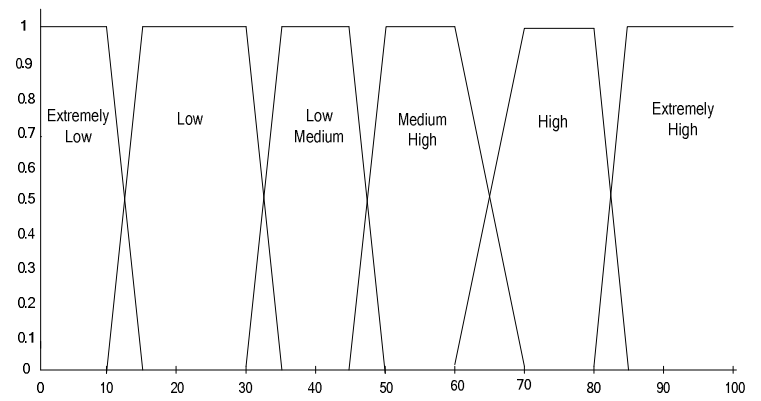
X-Axis showing the Magnitude of Possible Consequences of Failure in an Interaction
Y-Axis showing the Degree of Membership

Figure 2: Membership function of the input: Possible Consequences of Failure

The universe of discourse (UoD) for the LOIP in the interaction is $\{0,1,2,3,4,5 \ldots \ldots .100\}$; where each element has unit of \%. The input given by the risk assessing agent to the fuzzy system for the linguistic variable 'Loss of Investment Probability' is a crisp value within the range of 0-100. To transform the crisp value into a fuzzy value, we define six different fuzzy sets namely 'Extremely Low', 'Low', 'Low Medium', 'Medium High', 'High' and 'Extra High'. Within these different predicates, the degree of truth to which the input value of LOIP quantifies is determined by using the membership function. We define the membership function of the linguistic variable 'Loss of Investment Probability' in figure 3 .

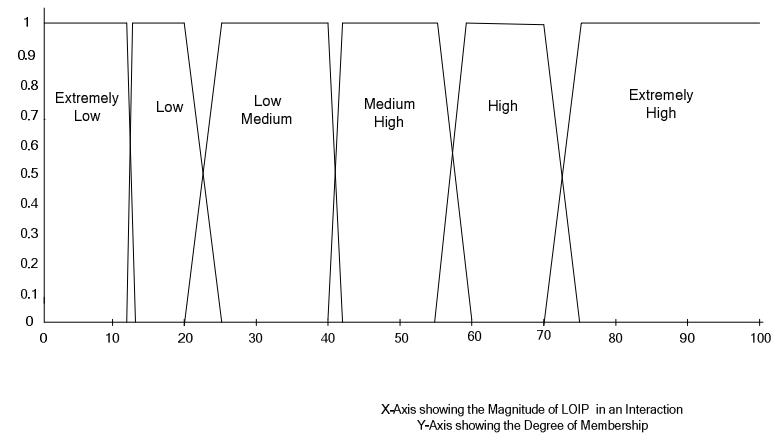

Figure 3: Membership function of the input: Loss of Investment Probability

The degree of membership (DOM) of a variable to a fuzzy set or predicate defines the magnitude of participation of that variable within that fuzzy set. The degree of membership for a variable is determined by plugging the selected input parameter into the horizontal axis of its UoD and projecting it vertically at the point of intersection with the fuzzy set / sets. The point on y-axis to which the input variable intersects a fuzzy set represents its degree of influence or the degree of membership (DOM) to that fuzzy set. Based on the membership functions, the focal elements of each input variable should be transformed into fuzzy sets, based on the level of truth to which the input variable quantifies to the predicates. To transform a focal element ' $x$ ' of an input linguistic variable to the defined fuzzy sets in it, the risk assessing agent has to determine the possibility to which that element ' $x$ ' corresponds with the defined predicates of that input variable, by considering the overlap between the degree of evidence of the input value ' $x$ ', with the degree of membership to which that input value ' $x$ ' corresponds to a particular predicate from the membership function for that input variable. The possibility that the fuzzy set or predicate ' $\mathrm{A}$ ' of an input linguistic variable will occur based on the degree of evidence of input ' $\mathrm{x}$ ' is given by [18]:

$$
\Pi(\mathrm{A})=\max \left\{\min \left[\pi(\mathrm{x}), \mathrm{DOM}_{\mathrm{A}}(\mathrm{x})\right]\right\}
$$

Equation 5 is repeated for each focal element ' $\mathrm{x}$ ' from the UoD for an input variable. Once the input variables have been transformed to their corresponding fuzzy sets, they must then be processed in the inference engine of the fuzzy system to draw conclusion on the UoD of the output linguistic variable, based on the given evidences on the fuzzy variables that it computes from the inputs. The fuzzy system based on the inputs given to it computes an output specifying the magnitude and level of perceived risk present in the interaction. The universe of discourse (UoD) of the output membership function 'Perceived Risk' is in the range of $\{0,1,2,3,4,5 \ldots \ldots 100\}$. We divide the UoD into six different fuzzy sets by using the predicates, 'Extremely Low', 'Low', 'Low Medium', 'Medium High', 'High' and 'Extremely High'. We define the membership function of the output 'Perceived Risk' in an interaction by using a trapezoidal curve as shown in figure 4:

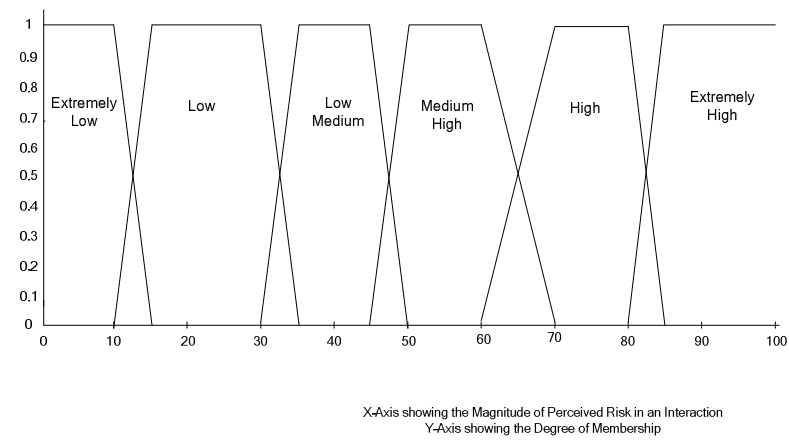

Figure 4: Membership function of the output: Perceived Risk in the Interaction

\section{DEFInING The RULes For The FUZZY MOdeL}

According to Mamdani approach after determining the possibility of the fuzzy sets of each input variable, they must be fed to the inference engine for drawing the conclusion or the output based on the given inputs. The inference engine consists of fuzzy rules by which the conclusions are made based on the given inputs. The rules which we use in our model are of the IF-THEN structure. We abbreviate the part between IF and THEN which refers to the conditional part of the rule as the 'LHS' (Left-hand side) of the rule. The LHS of the rules consists of the fuzzy sets of the inputs determined based on their focal elements. Similarly the impli- 
cation part of the rule, which is associated with the THEN operator, is abbreviated as the 'RHS' (Right-hand side) of the rule. The implication part of the rule gives the level of perceived risk in the interaction based on the computations performed by the inference engine, according to the inputs given to it. The LHS part of the rules is combined by a logic operator for the inference part to give its output. We use the AND operator as we want to consider the intersection between the input fuzzy sets, while implicating the magnitude of risk present in the interaction by utilizing those sets. In our fuzzy model, there are two inputs based on which the level of perceived risk is determined in the interaction. Each of the input is further defined by 6 predicates. Hence the total number of homogenous rules in our system will be: 6 $\mathrm{X} 6=36$. For ease in defining the rules we term the predicates of our fuzzy system by their acronyms and rules for the fuzzy model in table 1 and 2 respectively.

\begin{tabular}{|c|c|}
\hline Predicates or the Fuzzy sets & Corresponding Acronym \\
\hline Extremely Low & 'EL' \\
\hline Low & 'L' \\
\hline Low Medium & 'LM' \\
\hline Medium High & 'MH' \\
\hline High & 'H' \\
\hline Extremely High & 'EH' \\
\hline \multicolumn{2}{|c|}{ Table 1 acronyms of the predicates } \\
\hline
\end{tabular}

\begin{tabular}{|c|c|c|c|c|c|}
\hline & PCF & & LOIP & & PR \\
\hline If & EL & and & EL & then & L \\
\hline If & L & and & EL & then & L \\
\hline If & LM & and & EL & then & LM \\
\hline If & MH & and & EL & then & MH \\
\hline If & H & and & EL & then & H \\
\hline If & EH & and & EL & then & EH \\
\hline If & EL & and & L & then & L \\
\hline If & L & and & L & then & LM \\
\hline If & LM & and & L & then & MH \\
\hline If & MH & and & L & then & H \\
\hline If & H & and & L & then & EH \\
\hline If & EH & and & L & then & EH \\
\hline If & EL & and & LM & then & LM \\
\hline If & L & and & LM & then & MH \\
\hline If & LM & and & LM & then & H \\
\hline If & MH & and & LM & then & EH \\
\hline If & $\mathrm{H}$ & and & LM & then & EH \\
\hline If & EH & and & LM & then & EH \\
\hline If & EL & and & MH & then & MH \\
\hline If & L & and & MH & then & H \\
\hline If & LM & and & MH & then & H \\
\hline If & MH & and & MH & then & EH \\
\hline If & $\mathrm{H}$ & and & MH & then & EH \\
\hline
\end{tabular}

\begin{tabular}{|c|c|c|c|c|c|}
\hline If & EH & and & MH & then & EH \\
\hline If & EL & and & H & then & H \\
\hline If & L & and & H & then & H \\
\hline If & LM & and & H & then & EH \\
\hline If & MH & and & H & then & EH \\
\hline If & H & and & H & then & EH \\
\hline If & EH & and & H & then & EH \\
\hline If & EL & and & EH & then & EH \\
\hline If & L & and & EH & then & EH \\
\hline If & LM & and & EH & then & EH \\
\hline If & MH & and & EH & then & EH \\
\hline If & $\mathrm{H}$ & and & EH & then & EH \\
\hline If & EH & and & EH & then & EH \\
\hline
\end{tabular}

Table 2 defining the rules of the fuzzy system

The output of the fuzzy system will be determined by the degree or strength to which each rule fires. The rules use the possibility of the fuzzy set of each input as the weighting factor to determine their influence on the output fuzzy sets. Based on the possibility of each fuzzy set of the inputs, the RHS of the rules must be computed to determine the strength by which each rule fires. The output of each rule must be aggregated to determine the output fuzzy sets. The aggregation operator combines the output of the various rules corresponding to a fuzzy set into one. In our approach we will use the multiple or additive aggregation operator to consider all the consequent part of the rules which produces an output that relates to the same fuzzy set. The multiple additive method determines the strength to which the output fuzzy set quantifies, by considering all the rules which produce the same fuzzy set and then utilizes a fuzzy additive principal bounded to a sum of 1.0. This is in contrast to the Mamdani approach which considers all the rules which produce the same fuzzy set and then selects the maximum value from the rules as the strength to which that output fuzzy set quantifies. The aggregation process gives the output fuzzy sets to which the perceived risk in an interaction corresponds to along with the possibility of them occurring on the output membership function. These fuzzy sets represent the linguistic level of perceived risk along with their possibility of occurrence in the interaction. The risk assessing agent by analyzing the magnitude of perceived risk in the interaction numerically and linguistically can make an informed interacting based decision of its future interaction with a risk assessed agent.

\section{CONCLUSION}

In paper we proposed a methodology by which the risk assessing agent can combine the different constituents that it had determined for risk analysis and ascertain the perceived risk in interacting with a risk assessed agent. The methodology that we propose determines the perceived risk in an interaction numerically in terms of levels and linguistically in terms of semantics. The numerical representation of perceived risk utilizes possibility theory in determining the magnitude of perceived risk and the possibility of occurrence of those magnitudes. Linguistic representation of per- 
ceived risk utilizes fuzzy sets theory to determine in semantic terms the level of perceived risk in the interaction along with its possibility of occurrence. The risk assessing agent by ascertaining the perceived risk in the interaction numerically and linguistically can:

- Determine the magnitude of different level of perceived risk that could be present in the interaction,

- Determine the possibility of occurrence of each of those different numerical magnitudes of perceived risk,

- Semantically ascertain the perceived risk in an interaction in linguistic terms,

- Determine the possibility of occurrence of those semantic levels of perceived risk, and

- Alleviate the ambiguity and vagueness in its mind while determining the perceived risk in interacting with a risk assessed agent.

\section{REFERENCES}

[1] Elizabeth Chang, Tharam Dillon, and F. K. Hussain, Trust and Reputation for Service-Oriented Environments. West Sussex, England: John Wiley \& Sons, Ltd, 2006.

[2] Australian Bureau of Statistics, "Online Shopping Blooms," in 8147.0Use of the Internet by Householders, Australia, Nov 2000, 2000.

[3] L. Enos, "E-tail Invades the Real World," in E-Commerce Times, 2001 .

[4] Reineke Reitsma, "European Online Shopping Landscape 2007," in Forrester Research, 2007.

[5] Thuiswinkel Organisatie, "Internet shopping gaining popularity in the Netherlands," in $m \& c, 2007$.

[6] Wonseok Oh, "C2C Versus B2C: A Comparison of the Winner's Curse in Two Types of Electronic Auctions," International Journal of Electronic Commerce vol. Volume 6, pp. 115 - 138, Summer 20022002.

[7] J. Carter and A. A. Ghorbani, "Towards a formalization of Trust," Web Intelligence and Agent Systems, vol. Vol. 2, , pp. pp. 167-183., 2004.

[8] Yan Wang and F.-r. Lin, "Trust and Risk Evaluation of Transactions with Different Amounts in Peer-to-Peer E-commerce Environments," in IEEE International Conference on e-Business Engineering (ICEBE'06), Shanghai, 2006, pp. 102-109.

[9] David Gefen, V. Srinivasan Rao, and N. Tractinsky, "The Conceptualization of Trust, Risk and Their Relationship in Electronic Commerce: The Need for Clarifications," in Proceedings of the 36th Hawaii International Conference on System Sciences, Big Island, HI, USA, 2003, pp. 192-201.

[10] C. Cheung and M.K.O. Lee, "Trust in Internet shopping: A proposed model and measurement instrument, ," in Proceedings of the 6th Americans Conference on Information Systems, , 2000, pp. pp 681-689.

[11] D.M. Rousseau, S.B. Sitkin, R.S. Burt, and C. Camerer, " Not so different after all: A cross-discipline view of trust, ," Academy of Management Review, , vol. vol. 23, , pp. pp. 391-404., 1998.

[12] J.G. March and Z. Shapira, "Managerial perspective on risk and risk taking " Management Science, , vol. vol. 33, pp. 1404-1418, 1987.

[13] N. Luhmann, "Familiarity, confidence, trust: Problems and alternatives," in Making and Breaking Cooperative Relations New York, USA: Basil Blackwell, 1988, pp. 94-107.

[14] Sander Greenland, "Bounding Analysis as an Inadequately Specified Methodology," Risk Analysis, vol. 24, pp. 1085-1092, 2004.

[15] Omar Khadeer Hussain, Elizabeth Chang, Farookh Khadeer Hussain, and T. S. Dillon, "A methodology to quantify failure for risk-based decision support system in digital business ecosystems," Data and Knowledge Engineering, vol. Volume 63, pp. 597-621, 2007.

[16] Omar Khadeer Hussain, Elizabeth Chang, Farookh Khadeer Hussain, and T. S. Dillon, "Towards Quantifying the Possible Risk in ecommerce interactions for RDSS," in Proceedings of the IEEE International Conference on e-Business Engineering (ICEBE'07), Hong Kong, 2007, pp. 89-96

[17] Didier Dubious, Henri Prade, Henri Farreny, Roger MartinClouaire, Claudette Testemale, and E. F. Harding, Possibility Theory: An Approach to Computeraized Processing of Uncertainity. New York: Plenium Press, 1988.

[18] Riza C. Berkan and S. L. Trubatch, Fuzzy Systems Design Priciples. New York: IEEE Press, 1997. 